\title{
BIOMASS OF Eucalyptus globulus IN YOUNG PLANTATIONS IN URUGUAY
}

\author{
Óscar Vallejos-Barra ${ }^{1 *}$, Alfredo Ibáñez², Guillermo Morás³, Gustavo Daniluk-Mosquera ${ }^{4}$, \\ Mauricio Ponce-Donoso ${ }^{5}$
}

\author{
${ }^{1 *}$ Universidad de Talca, Facultad de Ciencias Forestales, Talca, Chile - e-mail: ovallejo@utalca.cl \\ ${ }^{2}$ Universidad de Talca, Centro Tecnológico de Hidrología Ambiental, Talca, Chile - e-mail: alibanez@utalca.cl \\ ${ }^{3}$ Universidad de la República, Departamento de Producción Forestal y Tecnología de la Madera, Montevideo, Uruguay- e-mail: \\ gmoras@fagro.edu.uy \\ ${ }^{4}$ Universidad de la República, Departamento de Producción Forestal y Tecnología de la Madera, Montevideo, Uruguay - e-mail: \\ gdaniluk@fagro.edu.uy \\ ${ }^{5}$ Universidad de Talca, Facultad de Ciencias Forestales, Talca, Chile - e-mail: mponce@utalca.cl
}

Received for publication: 03/04/2019 - Accepted for publication: 08/08/2019

\begin{abstract}
Resumo
Biomassa de Eucalyptus globulus em plantações jovens de Uruguai. Em quatro locais da costa oeste da República Oriental do Uruguai (Algorta, Bequeló, Quebracho e Tres Bocas) quantificou-se a biomassa aérea de plantações de Eucalyptus globulus com idades entre 3 e 9 anos. Em 20 parcelas de amostragem de $300 \mathrm{~m}^{2}$ 616 árvores foram medidas, selecionando 132 para a quantificação. Em cada árvore foi calculada a biomassa de cinco frações: folhas, galhos verdes, galhos secos, fuste e total aéreo, este último obtido pela adição das frações anteriores. Quatro funções alométricas foram ajustados em três cenários: por local, para o total de locais e para o total de locais, mas identificadas mediante variáveis binária. As funções por local apresentaram a mínima diferença do critério de informação de Akaike (AIC), de modo que em todos os casos foram selecionadas esse tipo de funções. Estimou-se a biomassa por hectare e o incremento médio anual, com valores médios para os locais $1,18 \pm 0,45,1,08 \pm 0,18,0,58 \pm 0,22,11,24 \pm 1,86$ e $14,20 \pm 2,50 \mathrm{Mg} \mathrm{ha}^{-1}$ ano $^{-1}$ para folhas, galhos verdes, galhos secos, fuste e total aéreo, respectivamente. Através de uma análise de variância por componente foram identificadas diferenças estatisticamente significativas da biomassa anual média entre os locais. Os maiores incrementos foram observados em Bequeló e Tres Bocas, seguido de Quebracho e Algorta. A análise variância incorporou como covariável a densidade da plantação, que resultou ser significativa nos componentes de maiores dimensões.

Palavras-chave: funções alométricas, variáveis binárias, critério de informação de Akaike.
\end{abstract}

\begin{abstract}
In four areas of the western coast of the Oriental Republic of Uruguay (Algorta, Bequeló, Quebracho and Tres Bocas) the aerial biomass of Eucalyptus globulus plantations was quantified with ages between 3 and 9 years. In 20 circular plots of $300 \mathrm{~m}^{2}$, 616 trees were measured, selecting 132 for quantification. In each tree, the biomass of five components was calculated: leaves, green branches, dry branches, stem and total aerial, the latter obtained by adding the previous components. Four allometric functions were adjusted in three scenarios: by location, for total locations and for total locations, but identified by dummy variables. The functions by location presented minimal difference from Akaike information criterion (AIC), so that in all cases this type of function was selected. The average annual increase in biomass was obtained, with average values for all the localities of $1.18 \pm 0.45,1.08 \pm 0.18,0.58 \pm 0.22,11.24 \pm 1.86$ y $14.20 \pm 2.50 \mathrm{Mg} \mathrm{ha}^{-1}$ year $^{-1}$ for leaves, green branches, dry branches, stem and total aerial, respectively. An analysis of variance by component identified statistically significant differences in the average annual increase in biomass between locations. The localities with the highest increase were Bequeló and Tres Bocas, followed by Quebracho and Algorta. The analysis of variance incorporated planting density as a covariate, proving to be significant for the components of greater dimensions.

Keywords: allometric functions, dummy variables, Akaike information criterion.
\end{abstract}

\section{INTRODUCTION}

In Uruguay $64 \%$ of wood production is used for energy purposes, with Eucalyptus globulus being the species with the largest forested area (DGF-MGAP, 2012). The estimation of aerial biomass from these plantations will serve to better characterize this resource, since the measurement units for these products are usually associated with biomass.

Tree biomass is often determined using destructive sampling (PÉREZ-CRUZADO and RODRÍGUEZSOALLEIRO, 2011), which according to Gibbs et al. (2007) is summarized in three steps, (i) obtaining the green mass of each component, (ii) taking a sample of the components and determining their green and dry mass, and

FLORESTA, Curitiba, PR, v. 50, n. 4, p. 1798 - 1807, out/dez 2020.

Vallejos-Barra, O. et.al.

ISSN eletrônico 1982-4688 
(iii) obtaining the dry mass of each component using proportions (Dry mass $=$ Green mass * Dry mass sample $/$ Green mass sample).

The allometric function (biomass $=b_{0} d^{b_{1}}$ ) is internationally the most widely used to determine tree biomass (ANTÓNIO et al., 2007; ZIANIS et al., 2011; KUYAH et al., 2013; PAUL et al., 2013). The coefficient $b_{1}$, called the universal exponent or allometric constant, should have a value of 8/3 (PILLI et al., 2006), although some studies have found lower values (ZIANIS and MENCUCCINI, 2004; PILLI et al., 2006; KUYAH et al., 2013). According to Wang et al. (2011) other variables such as tree height have been incorporated to improve biomass estimation (ANTÓNIO et al., 2007; ZEWDIE et al., 2009; ÁLVAREZ et al., 2012); however, their predictive ability is reduced (MONTAGU et al., 2005; PÉREZ-CRUZADO and RODRÍGUEZ-SOALLEIRO, 2011; KUYAH et al., 2012 and 2013). The density of the wood has also been incorporated achieving significant adjustments (KUYAH et al., 2012; ÁLVAREZ et al., 2012), although not in every case (KUYAH et al., 2013).

In general, biomass functions are adjusted by location for each of the considered components, or for the set of locations. In general, the functions for the whole have a lower performance than the functions by location (van BREUGEL et al., 2011; PAUL et al., 2013; VALLEJOS-BARRA et al.; 2014) and in some cases have variable results since in some locations there is little error, while in others the errors can be significant. (CHAVE et al., 2005).

Another alternative is to consider the set of localities but identifying each one of them by means of Dummy variables. This increases the degrees of freedom for the estimation of population parameters and therefore improves the relative precision of the estimates, which could not be verified by Vallejos-Barra et al. (2014); However, António et al. (2007) and Zeng et al. (2011) report that biomass estimation was improved by incorporating a Dummy variable identifying the origin of the stands.

Akaike Information Criterion (AIC) was used to select functions, since it is a powerful tool for comparing functions (SNIPES and TAYLOR, 2014). Burnham and Anderson (2002) describe it as:

$$
A I C=n \log \left(\hat{\sigma}^{2}\right)+2 K
$$

Where AIC is the Akaike Information Criterion, $\mathrm{n}$ is the amount of data used in the adjustment, $\hat{\sigma}^{2}=$ $\sum\left(y_{i}-\hat{y}_{i}\right)^{2} / n$ and $\mathrm{k}$ is the total of estimated parameters, including the intercept and $\hat{\sigma}^{2}$.

The AIC value measures loss of information by a given function (MAZEROLLE, 2006), but by itself its value is not relevant. The important thing is to obtain the difference of the AIC between each adjusted function and the function with the lowest value (BURNHAM and ANDERSON, 2002); so the best function will have a difference equal to zero; with a difference up to 2 , the function could be equally satisfactory. A difference between 4 and 7 reduces the significance of the function, while a difference greater than 10 rules out the use of the function.

The hypothesis is that it is possible to model the biomass of different components of E. globulus by means of allometric functions, it is also hypothesized that there should be no difference in the average annual increase between the sampled localities. The aim of this study was to select biomass functions for the leaves, green branches, dry branches, stem and total aerial, of E. globulus trees growing in the western coast of the Oriental Republic of Uruguay in South America, in order to estimate the average annual increase of the aerial biomass (Mg $\mathrm{ha}^{-1}$ year $^{-1}$ ) of each of the components and to observe if there are significant differences between the four locations sampled.

\section{MATERIALS AND METHODS}

In the sedimentary basin of the west coast of the Oriental Republic of Uruguay, the company EUFORES has unmanaged plantations of Eucalyptus globulus L. According to the Köppen-Geiger climate classification, the region is characterized by a temperate and humid climate with hot summers (Cfa) (INIA, 2011). Cruz et al. (2000) characterize this zone with an average annual rainfall of $1,174 \mathrm{~mm}$ and average monthly temperatures ranging from 10.5 to $22.5^{\circ} \mathrm{C}$. The relief is of high and medium plains with soft, sometimes flattened hills and strong hills, which are no higher than one hundred meters above sea level.

The measurements were taken on 20 circular plots of $300 \mathrm{~m}^{2}$ in 4 geographical locations (6 in Algorta, 6 in Bequeló, 6 in Quebracho and 2 in Tres Bocas) where EUFORES has young plantations. The planting density varied between 733 and 1267 trees ha ${ }^{-1}$ with ages between 3 and 9 years. The plots were representative of the average growth of trees in each locality, based on data from permanent plots of the owner company.

In the plots the diameter at breast height (DBH) of all trees was recorded (616 in total), with a calliper of parallel arms, graduated to the millimeter. A total of 132 trees representative of the measured diameter classes were upturned (40 in Algorta, 42 in Bequeló, 36 in Quebracho and 14 in Tres Bocas). The height of the trees was obtained by measuring the length of each one of them, once they were turned over, using a distance measuring device, graduated to the millimeter. Determining height in this way is common in some studies (ÁLVAREZ et al., 2012; KUYAH et al., 2013). Each tree was divided into four components: green branches, dry branches, leaves and stem. The branches were separated from the stem with an axe or pruning shears when their size allowed. The

FLORESTA, Curitiba, PR, v. 50, n. 4, p. 1798 - 1807, out/dez 2020.

Vallejos-Barra, O. et.al

ISSN eletrônico 1982-4688

DOI: $10.5380 /$ rf.v50 i4. 65781 
leaves were manually separated from the branches and the stem was cut into two-meter-long logs. Additionally, the diameters of each of the logs were recorded, in order to calculate the volume of the stem, by adding the Smalian volume of each of the logs.

In the field, the green mass of the components was obtained using a Thunderbird field balance with an accuracy of $200 \mathrm{~g}$. A sample of each component was sent to the laboratory recording the green mass with a My Weigh KD 600 electronic balance with an accuracy of $1 \mathrm{~g}$. Later, when the samples reached a constant mass in a Venticell oven at $65^{\circ} \mathrm{C}$, their dry mass was obtained. The dry mass of each tree component was calculated by multiplying the ratio between the dry and green mass of the sample obtained in the laboratory by the green mass recorded in the field. The fifth component, the total aerial dry mass of the tree, resulted from the addition of the dry mass of the components recorded in the field. The basic density of the stem wood was obtained by dividing the dry mass of the stem by the volume of the latter.

The entire statistical analysis was performed with the free statistical program $\mathrm{R}$ version 3.4.0. Four nonlinear functions ([1] to [4]) were adjusted for each component in each of the locations. The non-linear adjustment method (nls), which uses the Gauss-Newton method, was used for regression adjustment.

$$
\begin{aligned}
& B=b_{0} d^{b_{1}} \\
& B=b_{0} d^{b_{1}} \rho \\
& B=b_{0} d^{b_{1}} h^{b_{2}} \\
& B=b_{0} d^{b_{1}} h^{b_{2}} \rho
\end{aligned}
$$

Where B is the biomass of each component $(\mathrm{kg}), \mathrm{d}$ is the diameter at breast height $(\mathrm{cm}), \mathrm{h}$ is the total height $(\mathrm{m}), \rho$ is the basic density of the stem $\left(\mathrm{kg} \mathrm{m}^{-3}\right), b_{0}$ is the regression constant and $b_{i}$ are the regression coefficients $(i=1$ and 2$)$.

With these same functions ([1] to [4]) an adjustment was made for the total number of locations, which considered the data from all locations together. Finally, another adjustment was made for total locations, with four non-linear functions ([5] to [8]), where each location was identified by dummy variables.

$$
\begin{aligned}
& B=\left(b_{0} L 1+b_{1} L 2+b_{2} L 3+b_{3} L 4\right) d^{\left(b_{4} L 1+b_{5} L 2+b_{6} L 3+b_{7} L 4\right)} \\
& B=\left(b_{0} L 1+b_{1} L 2+b_{2} L 3+b_{3} L 4\right) d^{\left(b_{4} L 1+b_{5} L 2+b_{6} L 3+b_{7} L 4\right)} \rho \\
& B=\left(b_{0} L 1+b_{1} L 2+b_{2} L 3+b_{3} L 4\right) d^{\left(b_{4} L 1+b_{5} L 2+b_{6} L 3+b_{7} L 4\right)} h^{\left(b_{8} L 1+b_{9} L 2+b_{10} L 3+b_{11} L 4\right)} \\
& B=\left(b_{0} L 1+b_{1} L 2+b_{2} L 3+b_{3} L 4\right) d^{\left(b_{4} L 1+b_{5} L 2+b_{6} L 3+b_{7} L 4\right)} h^{\left(b_{8} L 1+b_{9} L 2+b_{10} L 3+b_{11} L 4\right)} \rho
\end{aligned}
$$

Where $b_{j}(j=0,1, \ldots, 3)$ are the regression constants, $b_{k}(k=4,5, \ldots, 11)$ are the regression coefficients and $L_{m}(m=1,2, \ldots, 4)$ are the binary variables that identify each location. $\mathrm{L}_{1}$ is 1 if the locations is Algorta and 0 in any other case, $\mathrm{L}_{2}$ is 1 when considering the location Bequeló and 0 in any other case, $\mathrm{L}_{3}$ is 1 if the location is Quebracho and 0 in any other case and $\mathrm{L}_{4}$ is 1 when considering the location Tres Bocas and 0 in any other case.

A total of 120 function settings were made: 80 function settings per location ( 5 components $* 4$ locations $* 4$ functions per location) and 40 global settings ( 5 components * ( 4 global functions +4 global dummy functions). In order to make the adjustments of the various functions comparable, the Akaike information criterion (AIC) was calculated by component and for each location, then the differences were obtained in relation to the lowest AIC, as recommended by Burnham and Anderson (2002) and Burnham et al. (2011); selecting those functions with the minimum values of this difference. As some of the selected functions incorporated the height of the trees, functions ([9] to [11]) were adjusted to estimate this variable in those trees where it was not measured. An adjustment was made for each location and another for all locations, so that a total of 15 function adjustments were made.

$$
\begin{aligned}
& h=b_{0} d^{b_{1}} \\
& h=b_{0} \exp \left(b_{1} / d\right) \\
& h=b 0+b 1 d
\end{aligned}
$$

The regression adjustment for stem density was not significant so the probability density function that best represented the basic stem density was determined, with the intention of giving readers the inverse integral to estimate the density at a certain probability. With the selected functions, the biomass of each of the plots was estimated and the existence of statistically significant differences in the increase of biomass per hectare between

FLORESTA, Curitiba, PR, v. 50, n. 4, p. 1798 - 1807, out/dez 2020. 
the localities was determined using an analysis of parametric variance. The analyzed variable was the average annual increase of biomass per hectare (MAI Mg B ha ${ }^{-1}$ ) and as there was variation in planting density, the number of trees $\mathrm{ha}^{-1}$ from each plot was included as a covariate. The assumptions of this analysis were verified with the Shapiro-Wilk and Levene tests. When there were statistically significant differences between the sources of variation these were identified with Duncan's multiple comparison test.

\section{RESULTS}

The mean annual increment (MAI) in diameter at breast height and height was used to characterize each locality (Table 1). Both increases diminished as the age of the plots increased. Over 5 years of age, regardless of

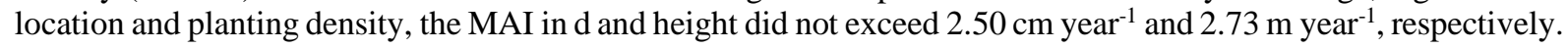
The largest increases were obtained in Bequeló up to 5 years of age. The increase was equal to or greater than 3.37 $\mathrm{cm} \mathrm{year}^{-1}$ and $3.02 \mathrm{~m} \mathrm{year}^{-1}$, for the diameter at breast height and the height, respectively. These increases are associated with lower planting densities $\left(\leq 1034\right.$ trees $\left.^{-1} \mathrm{a}^{-1}\right)$. No clear trend was observed in the decrease or increase of the MAI in diameter at breast height and height with fluctuation in the number of trees ha-1.

Table 1. Dendrometric description of locations

Tabela 1. Descrição dendrométricas dos locais

\begin{tabular}{cccccc}
\hline Location & $\begin{array}{c}\text { Age } \\
(\text { years })\end{array}$ & $\begin{array}{c}\text { MAI d } \\
\left(\mathbf{c m ~ y e a r}^{-\mathbf{1}}\right)\end{array}$ & $\begin{array}{c}\text { MAI h } \\
\left(\mathbf{m ~ y e a r}^{-\mathbf{1}}\right)\end{array}$ & $\begin{array}{c}\text { Trees ha } \\
\text { average }^{-1}\end{array}$ & $\begin{array}{c}\boldsymbol{\rho}_{\text {average }} \\
\left(\mathbf{k g ~ m}^{-3}\right)\end{array}$ \\
\hline Algorta & 4 & 2.92 & 3.11 & 1,267 & 485.5 \\
& 5 & 2.41 & 2.51 & 1,034 & 482.1 \\
\hline Bequeló & 8 & 1.76 & 1.93 & 1,033 & 530.4 \\
& 3 & 4.36 & 3.85 & 833 & 496.2 \\
& 5 & 3.36 & 3.26 & 1,034 & 488.4 \\
\hline Quebracho & 6 & 3.37 & 3.02 & 733 & 518.2 \\
\hline Tres Bocas & 8 & 2.50 & 2.73 & 1,233 & 537.0 \\
\hline
\end{tabular}

Source: Vallejos-Barra, et al (2014), available at < https://www.ipef.br/publicacoes/scientia/nr101/cap01.pdf >

300 Akaike information criteria (AIC) were calculated, which were transformed into the same amount of differences of AIC ( 
Table 2). The number of AICs (300) exceeded the number of adjustments (120), since the AIC was obtained by location, function and component (240 AIC) and also by function and component for all locations (60 AIC).

It is observed that the differences equal to zero are only found in the functions adjusted for each of the locations. Both adjustments for the total number of locations, in their great majority, were not significant since the differences were greater than 10 .

The selected functions, those with a difference equal to zero, are presented in

\begin{tabular}{|c|c|c|c|c|c|c|c|c|c|c|c|}
\hline \multirow[b]{2}{*}{ 苞 } & \multirow[b]{2}{*}{ } & \multicolumn{5}{|c|}{ Adjustment by location } & \multicolumn{5}{|c|}{ Adjustment for total locations } \\
\hline & & 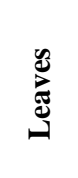 & 总 & 莺 & 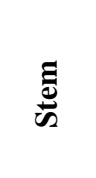 & 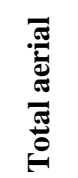 & 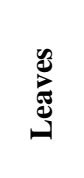 & صِّ & 党 & $\underset{\mathscr{D}}{E}$ & 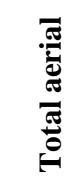 \\
\hline 1 & \multirow{8}{*}{$\begin{array}{l}\frac{\pi}{0} \\
\frac{0}{2} \\
\frac{\infty}{Z}\end{array}$} & 0.0 & 0.0 & 0.0 & 22.8 & 25.5 & 4.0 & 2.8 & 12.4 & 23.7 & 27.7 \\
\hline 2 & & 4.6 & 0.8 & 1.7 & 7.3 & 5.3 & 6.0 & 2.1 & 14.8 & 8.2 & 5.9 \\
\hline 3 & & 1.8 & 2.0 & 1.6 & 0.1 & 0.2 & 18.9 & 6.4 & 14.5 & 7.1 & 5.0 \\
\hline 4 & & 6.2 & 2.0 & 3.6 & 0.0 & 0.0 & 19.2 & 4.8 & 16.0 & 16.2 & 14.2 \\
\hline 5 & & & & & & & 12.0 & 12.0 & 12.0 & 34.8 & 37.5 \\
\hline 6 & & & & & & & 16.6 & 12.8 & 13.7 & 19.3 & 17.3 \\
\hline 7 & & & & & & & 19.8 & 20.0 & 19.6 & 18.0 & 18.0 \\
\hline 8 & & & & & & & 24.2 & 20.0 & 21.6 & 18.1 & 18.2 \\
\hline 1 & \multirow{8}{*}{ 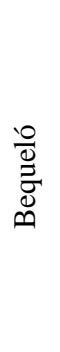 } & 23.9 & 20.8 & 0.0 & 68.4 & 28.5 & 28.3 & 24.9 & 5.3 & 93.8 & 57.2 \\
\hline 2 & & 25.2 & 18.3 & 6.0 & 56.2 & 9.4 & 29.6 & 22.2 & 10.5 & 85.4 & 46.3 \\
\hline 3 & & 3.4 & 7.8 & 1.6 & 27.8 & 20.0 & 20.0 & 25.3 & 8.4 & 43.4 & 35.7 \\
\hline 4 & & 0.0 & 0.0 & 7.2 & 0.0 & 0.0 & 16.1 & 20.5 & 14.1 & 29.2 & 23.8 \\
\hline 5 & & & & & & & 35.9 & 32.8 & 12.0 & 80.4 & 40.5 \\
\hline 6 & & & & & & & 37.2 & 30.3 & 18.0 & 68.2 & 21.4 \\
\hline 7 & & & & & & & 21.4 & 25.8 & 19.6 & 45.8 & 38.0 \\
\hline 8 & & & & & & & 18.0 & 18.0 & 25.2 & 18.0 & 18.0 \\
\hline 1 & \multirow{8}{*}{$\begin{array}{l}\frac{8}{0} \\
\frac{0}{0} \\
0 \\
0 \\
0 \\
0\end{array}$} & 0.0 & 14.1 & 10.7 & 29.3 & 18.8 & 5.9 & 19.5 & 27.8 & 63.1 & 45.1 \\
\hline 2 & & 0.6 & 11.0 & 5.5 & 19.2 & 0.9 & 9.4 & 15.1 & 26.5 & 43.3 & 19.8 \\
\hline 3 & & 1.3 & 1.9 & 3.4 & 28.0 & 20.6 & 4.7 & 21.5 & 27.3 & 44.7 & 38.1 \\
\hline 4 & & 2.4 & 0.0 & 0.0 & 0.0 & 0.0 & 8.1 & 16.6 & 24.3 & 6.9 & 7.2 \\
\hline 5 & & & & & & & 12.0 & 26.1 & 22.7 & 41.3 & 30.8 \\
\hline 6 & & & & & & & 12.6 & 23.0 & 17.5 & 31.2 & 12.9 \\
\hline 7 & & & & & & & 19.3 & 19.9 & 21.4 & 46.0 & 38.6 \\
\hline 8 & & & & & & & 20.4 & 18.0 & 18.0 & 18.0 & 18.0 \\
\hline 1 & \multirow{8}{*}{ 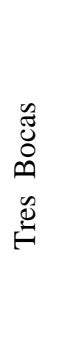 } & 3.0 & 1.6 & 0.1 & 17.0 & 12.9 & 6.5 & 12.4 & 1.7 & 30.5 & 28.2 \\
\hline 2 & & 0.0 & 0.0 & 0.0 & 12.3 & 4.3 & 2.7 & 9.6 & 1.0 & 24.7 & 20.5 \\
\hline 3 & & 4.6 & 2.6 & 1.4 & 8.7 & 10.8 & 9.1 & 14.7 & 3.9 & 16.5 & 20.2 \\
\hline 4 & & 1.4 & 0.4 & 1.3 & 0.0 & 0.0 & 5.8 & 12.4 & 3.3 & 8.1 & 10.8 \\
\hline 5 & & & & & & & 15.0 & 13.6 & 12.1 & 29.0 & 24.9 \\
\hline 6 & & & & & & & 12.0 & 12.0 & 12.0 & 24.3 & 16.3 \\
\hline 7 & & & & & & & 22.6 & 20.6 & 19.4 & 26.7 & 28.8 \\
\hline 8 & & & & & & & 19.4 & 18.4 & 19.3 & 18.0 & 18.0 \\
\hline 1 & \multirow{7}{*}{ 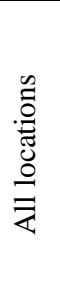 } & 41.5 & 48.0 & 6.2 & 141.6 & 94.3 & 58.3 & 71.5 & 41.7 & 215.2 & 176.7 \\
\hline 2 & & 44.5 & 40.4 & 9.2 & 98.4 & 28.5 & 60.4 & 59.4 & 46.1 & 172.3 & 118.4 \\
\hline 3 & & 4.4 & 13.7 & 0.0 & 50.6 & 53.9 & 49.5 & 72.6 & 42.7 & 97.1 & 107.5 \\
\hline 4 & & 0.0 & 0.0 & 5.0 & 0.0 & 0.0 & 42.2 & 57.5 & 45.4 & 62.8 & 61.7 \\
\hline 5 & & & & & & & 53.5 & 60.0 & 18.2 & 153.6 & 106.3 \\
\hline 6 & & & & & & & 56.5 & 52.5 & 21.2 & 110.4 & 40.5 \\
\hline 7 & & & & & & & 22.4 & 31.7 & 18.0 & 68.6 & 71.9 \\
\hline
\end{tabular}

FLORESTA, Curitiba, PR, v. 50, n. 4, p. 1798 - 1807, out/dez 2020. 
FLORESTA, Curitiba, PR, v. 50, n. 4, p. 1798 - 1807, out/dez 2020. 
Table 3) where the function [4] adjusted for each of the locations has the highest frequency.

Since function [4], selected for several components, incorporates the tree heights, functions ([9] to [11]) were adjusted to estimate the height in those trees where it was not measured.

Height per location has better adjustment indicators (Table 4), as happened with the biomass adjustments

FLORESTA, Curitiba, PR, v. 50, n. 4, p. 1798 - 1807, out/dez 2020. 
Table 2). In Table 5 the coefficients of selected functions are presented.

FLORESTA, Curitiba, PR, v. 50, n. 4, p. 1798 - 1807, out/dez 2020. 
Table 2. AIC differences in functions that estimate biomass according to function, component and location. Tabela 2. Diferença do AIC em funções que estimam a biomassa por função, componente e localidade.

\begin{tabular}{|c|c|c|c|c|c|c|c|c|c|c|c|}
\hline \multirow[b]{2}{*}{ 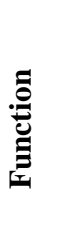 } & \multirow[b]{2}{*}{ } & \multicolumn{5}{|c|}{ Adjustment by location } & \multicolumn{5}{|c|}{ Adjustment for total locations } \\
\hline & & 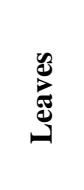 & 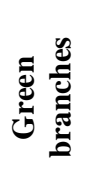 & 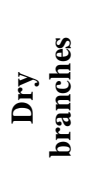 & $\stackrel{\Xi}{\infty}$ & & 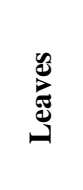 & 总 & 氖 & $\stackrel{\Xi}{\mathscr{D}}$ & \\
\hline 1 & \multirow{8}{*}{$\begin{array}{l}\frac{\pi}{0} \\
00 \\
\frac{00}{4}\end{array}$} & 0.0 & 0.0 & 0.0 & 22.8 & 25.5 & 4.0 & 2.8 & 12.4 & 23.7 & 27.7 \\
\hline 2 & & 4.6 & 0.8 & 1.7 & 7.3 & 5.3 & 6.0 & 2.1 & 14.8 & 8.2 & 5.9 \\
\hline 3 & & 1.8 & 2.0 & 1.6 & 0.1 & 0.2 & 18.9 & 6.4 & 14.5 & 7.1 & 5.0 \\
\hline 4 & & 6.2 & 2.0 & 3.6 & 0.0 & 0.0 & 19.2 & 4.8 & 16.0 & 16.2 & 14.2 \\
\hline 5 & & & & & & & 12.0 & 12.0 & 12.0 & 34.8 & 37.5 \\
\hline 6 & & & & & & & 16.6 & 12.8 & 13.7 & 19.3 & 17.3 \\
\hline 7 & & & & & & & 19.8 & 20.0 & 19.6 & 18.0 & 18.0 \\
\hline 8 & & & & & & & 24.2 & 20.0 & 21.6 & 18.1 & 18.2 \\
\hline 1 & \multirow{8}{*}{ 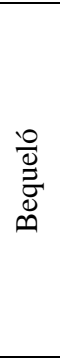 } & 23.9 & 20.8 & 0.0 & 68.4 & 28.5 & 28.3 & 24.9 & 5.3 & 93.8 & 57.2 \\
\hline 2 & & 25.2 & 18.3 & 6.0 & 56.2 & 9.4 & 29.6 & 22.2 & 10.5 & 85.4 & 46.3 \\
\hline 3 & & 3.4 & 7.8 & 1.6 & 27.8 & 20.0 & 20.0 & 25.3 & 8.4 & 43.4 & 35.7 \\
\hline 4 & & 0.0 & 0.0 & 7.2 & 0.0 & 0.0 & 16.1 & 20.5 & 14.1 & 29.2 & 23.8 \\
\hline 5 & & & & & & & 35.9 & 32.8 & 12.0 & 80.4 & 40.5 \\
\hline 6 & & & & & & & 37.2 & 30.3 & 18.0 & 68.2 & 21.4 \\
\hline 7 & & & & & & & 21.4 & 25.8 & 19.6 & 45.8 & 38.0 \\
\hline 8 & & & & & & & 18.0 & 18.0 & 25.2 & 18.0 & 18.0 \\
\hline 1 & \multirow{8}{*}{$\begin{array}{l}\frac{8}{0} \\
\frac{\pi}{0} \\
0 \\
0 \\
0\end{array}$} & 0.0 & 14.1 & 10.7 & 29.3 & 18.8 & 5.9 & 19.5 & 27.8 & 63.1 & 45.1 \\
\hline 2 & & 0.6 & 11.0 & 5.5 & 19.2 & 0.9 & 9.4 & 15.1 & 26.5 & 43.3 & 19.8 \\
\hline 3 & & 1.3 & 1.9 & 3.4 & 28.0 & 20.6 & 4.7 & 21.5 & 27.3 & 44.7 & 38.1 \\
\hline 4 & & 2.4 & 0.0 & 0.0 & 0.0 & 0.0 & 8.1 & 16.6 & 24.3 & 6.9 & 7.2 \\
\hline 5 & & & & & & & 12.0 & 26.1 & 22.7 & 41.3 & 30.8 \\
\hline 6 & & & & & & & 12.6 & 23.0 & 17.5 & 31.2 & 12.9 \\
\hline 7 & & & & & & & 19.3 & 19.9 & 21.4 & 46.0 & 38.6 \\
\hline 8 & & & & & & & 20.4 & 18.0 & 18.0 & 18.0 & 18.0 \\
\hline 1 & \multirow{8}{*}{ 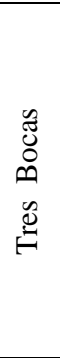 } & 3.0 & 1.6 & 0.1 & 17.0 & 12.9 & 6.5 & 12.4 & 1.7 & 30.5 & 28.2 \\
\hline 2 & & 0.0 & 0.0 & 0.0 & 12.3 & 4.3 & 2.7 & 9.6 & 1.0 & 24.7 & 20.5 \\
\hline 3 & & 4.6 & 2.6 & 1.4 & 8.7 & 10.8 & 9.1 & 14.7 & 3.9 & 16.5 & 20.2 \\
\hline 4 & & 1.4 & 0.4 & 1.3 & 0.0 & 0.0 & 5.8 & 12.4 & 3.3 & 8.1 & 10.8 \\
\hline 5 & & & & & & & 15.0 & 13.6 & 12.1 & 29.0 & 24.9 \\
\hline 6 & & & & & & & 12.0 & 12.0 & 12.0 & 24.3 & 16.3 \\
\hline 7 & & & & & & & 22.6 & 20.6 & 19.4 & 26.7 & 28.8 \\
\hline 8 & & & & & & & 19.4 & 18.4 & 19.3 & 18.0 & 18.0 \\
\hline 1 & \multirow{8}{*}{ 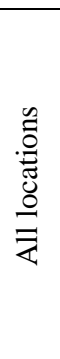 } & 41.5 & 48.0 & 6.2 & 141.6 & 94.3 & 58.3 & 71.5 & 41.7 & 215.2 & 176.7 \\
\hline 2 & & 44.5 & 40.4 & 9.2 & 98.4 & 28.5 & 60.4 & 59.4 & 46.1 & 172.3 & 118.4 \\
\hline 3 & & 4.4 & 13.7 & 0.0 & 50.6 & 53.9 & 49.5 & 72.6 & 42.7 & 97.1 & 107.5 \\
\hline 4 & & 0.0 & 0.0 & 5.0 & 0.0 & 0.0 & 42.2 & 57.5 & 45.4 & 62.8 & 61.7 \\
\hline 5 & & & & & & & 53.5 & 60.0 & 18.2 & 153.6 & 106.3 \\
\hline 6 & & & & & & & 56.5 & 52.5 & 21.2 & 110.4 & 40.5 \\
\hline 7 & & & & & & & 22.4 & 31.7 & 18.0 & 68.6 & 71.9 \\
\hline 8 & & & & & & & 18.0 & 18.0 & 23.0 & 18.0 & 18.0 \\
\hline
\end{tabular}

FLORESTA, Curitiba, PR, v. 50, n. 4, p. 1798 - 1807, out/dez 2020. 
Table 3. Selected functions for biomass and adjustment indicators by component and location.

Tabela 3. Funções selecionadas para biomassa e indicadores do ajuste por componente e localidade.

\begin{tabular}{|c|c|c|c|c|c|c|c|c|c|c|c|}
\hline Component & Location & Function & $\mathbf{b}_{\mathbf{0}}$ & $\mathbf{b}_{1}$ & $\mathbf{b}_{2}$ & Sbo & $\mathbf{S b}_{1}$ & $\mathbf{S b}_{2}$ & EEE & $\mathbf{R}^{2} \mathbf{A}$ & AIC \\
\hline \multirow{4}{*}{ Leaves } & Algorta & [ 1 ] & $7.917 \mathrm{E}-3$ & 2.410 & - & $5.970 \mathrm{E}-3$ & 0.261 & - & 1.9 & 0.76 & 51.4 \\
\hline & Bequeló & [4] & $4.522 \mathrm{E}-4$ & 3.463 & -2.235 & $3.181 \mathrm{E}-4$ & 0.334 & 0.380 & 2.7 & 0.75 & 85.8 \\
\hline & Quebracho & [ 1 ] & $1.672 \mathrm{E}-2$ & 2.145 & - & $1.102 \mathrm{E}-2$ & 0.223 & - & 1.9 & 0.81 & 47.6 \\
\hline & Tres Bocas & [2] & $2.697 \mathrm{E}-6$ & 3.004 & - & $4.121 \mathrm{E}-6$ & 0.505 & - & 2.3 & 0.85 & 25.8 \\
\hline \multirow{4}{*}{$\begin{array}{c}\text { Green } \\
\text { branches }\end{array}$} & Algorta & [ 1 ] & $1.335 \mathrm{E}-2$ & 2.273 & - & $1.200 \mathrm{E}-2$ & 0.312 & - & 2.6 & 0.65 & 74.6 \\
\hline & Bequeló & [ 4 ] & $6.378 \mathrm{E}-5$ & 4.569 & -2.757 & $7.127 \mathrm{E}-5$ & 0.532 & 0.582 & 3.3 & 0.66 & 103.1 \\
\hline & Quebracho & [ 4 ] & $2.432 \mathrm{E}-3$ & 3.416 & -2.612 & $2.565 \mathrm{E}-3$ & 0.367 & 0.581 & 3.0 & 0.82 & 82.2 \\
\hline & Tres Bocas & [ 2 ] & $3.121 \mathrm{E}-8$ & 4.561 & - & $6.374 \mathrm{E}-8$ & 0.667 & - & 3.0 & 0.88 & 32.5 \\
\hline \multirow{4}{*}{$\begin{array}{c}\text { Dry } \\
\text { branches }\end{array}$} & Algorta & [ 1 ] & $6.139 \mathrm{E}-1$ & 0.682 & - & $3.713 \mathrm{E}-1$ & 0.223 & - & 1.5 & 0.25 & 34.3 \\
\hline & Bequeló & [ 1 ] & & & - & $1.066 \mathrm{E}-1$ & 0.257 & - & 1.2 & 0.33 & 15.3 \\
\hline & Quebracho & [ 4 ] & $1.283 \mathrm{E}-4$ & 3.572 & -2.187 & $1.964 \mathrm{E}-4$ & 0.436 & 0.772 & 1.0 & 0.79 & 2.1 \\
\hline & Tres Bocas & [2] & $3.138 \mathrm{E}-5$ & 1.900 & - & $7.477 \mathrm{E}-5$ & 0.801 & - & 2.1 & 0.46 & 22.1 \\
\hline \multirow{4}{*}{ Stem } & Algorta & {$[4]$} & $1.132 \mathrm{E}-4$ & 2.091 & 0.503 & $4.083 \mathrm{E}-5$ & 0.141 & 0.164 & 9.5 & 0.95 & 183.3 \\
\hline & Bequeló & [ 4 ] & $5.086 \mathrm{E}-5$ & 1.552 & 1.282 & $1.230 \mathrm{E}-5$ & 0.097 & 0.121 & 6.3 & 0.98 & 157.1 \\
\hline & Quebracho & [ 4] & $9.677 \mathrm{E}-5$ & 1.920 & 0.721 & $2.951 \mathrm{E}-5$ & 0.066 & 0.143 & 5.1 & 0.99 & 119.9 \\
\hline & Tres Bocas & [4] & $3.562 \mathrm{E}-6$ & 1.500 & 2.220 & $3.537 \mathrm{E}-6$ & 0.232 & 0.500 & 7.7 & 0.99 & 59.6 \\
\hline \multirow{4}{*}{$\begin{array}{c}\text { Aerial } \\
\text { total }\end{array}$} & Algorta & [4] & $2.149 \mathrm{E}-4$ & 2.130 & 0.307 & $5.839 \mathrm{E}-5$ & 0.106 & 0.120 & 9.0 & 0.97 & 178.9 \\
\hline & Bequeló & [4] & $1.844 \mathrm{E}-4$ & 1.939 & 0.508 & $5.385 \mathrm{E}-5$ & 0.119 & 0.146 & 9.8 & 0.96 & 194.3 \\
\hline & Quebracho & [4] & $2.538 \mathrm{E}-4$ & 2.105 & 0.277 & 8.904E-5 & 0.079 & 0.167 & 7.3 & 0.99 & 146.2 \\
\hline & Tres Bocas & [4] & $1.406 \mathrm{E}-5$ & 2.015 & 1.328 & $1.422 \mathrm{E}-5$ & 0.252 & 0.523 & 9.6 & 0.99 & 66.0 \\
\hline
\end{tabular}

Where $b_{0}$ is the regression constant, $b 1$ and $b 2$ are the regression coefficients, $S b_{i}$ is the standard error of the regression constant if $i=0$ and of the regression coefficients if $\mathrm{i}=1$ or 2 , EEE is the standard error of estimation in kilograms, $\mathrm{R}^{2} \mathrm{~A}$ is the adjusted coefficient of determination and AIC is Akaike information criterion.

Table 4. Differences of the AIC in functions that estimate the height according to function, component, and location.

Tabela 4. Diferença do AIC em funções que estimam a altura por função, componente e localidade.

\begin{tabular}{ccccccc}
\hline \multirow{2}{*}{ Function } & \multirow{2}{*}{ Adjustment } & \multicolumn{3}{c}{ By location } & \multirow{2}{*}{ All locations } \\
\cline { 3 - 6 } & & Algorta & Bequeló & Quebracho & Tres Bocas & \\
\hline 9 & \multirow{2}{*}{ Per location } & 0.0 & 0.0 & 12.8 & 3.2 & 2.4 \\
10 & & 4.5 & 0.9 & 0.0 & 6.0 & 16.3 \\
11 & & 1.1 & 0.6 & 25.1 & 20.5 & 61.8 \\
9 & & 6.8 & 16.0 & 35.3 & 22.5 & 71.2 \\
10 & \multirow{2}{*}{ For all locations } & 10.5 & 21.3 & 32.8 & 20.5 & 66.6 \\
\hline 11 & & 7.1 & 14.8 & 40.8 & & \\
\hline
\end{tabular}

Table 5. Selected functions for height and adjustment indicators according to location

Tabela 5. Funções selecionadas para altura e indicadores do ajuste por localidade.

\begin{tabular}{ccccccccc}
\hline Location & Function & $\mathbf{b}_{\mathbf{0}}$ & $\mathbf{b}_{\mathbf{1}}$ & $\mathbf{S b}_{\mathbf{0}}$ & $\mathbf{S b}_{\mathbf{1}}$ & $\mathbf{E E E}$ & $\mathbf{R}^{\mathbf{2}} \mathbf{A}$ & $\mathbf{A I C}$ \\
\hline Algorta & {$[9]$} & 3.522 & 0.536 & 0.649 & 0.0687 & 2.0 & 0.66 & 56.5 \\
Bequeló & {$[9]$} & 3.099 & 0.575 & 0.626 & 0.0737 & 1.9 & 0.60 & 53.8 \\
Quebracho & {$[10]$} & 26.448 & -5.816 & 0.957 & 0.484 & 1.4 & 0.89 & 24.3 \\
Tres Bocas & {$[10]$} & 31.907 & -8.107 & 1.904 & 0.933 & 1.3 & 0.90 & 7.8 \\
\hline
\end{tabular}

Where $b_{0}$ is the regression constant, $b_{1}$ is the regression coefficient, $S b_{i}$ is the standard error of the regression constant, if $i=0$ and of the regression coefficient if $\mathrm{i}=1$, EEE is the standard error of estimation in meters, $\mathrm{R}^{2} \mathrm{~A}$ is the adjusted coefficient of determination and AIC is the Akaike information criterion.

The biomass functions [2] and [4] require the estimation of the stem density, but this variable did not present a connection with the recorded variables, so it was decided to select the probability density function that 
represented this variable. Of the thirty functions tested, the Logistics function [12] was selected, which obtained a Kolmogorov-Smirnov test statistic of 0.044 , with a probability of 0.96 . To obtain the basic density of the stem at a specific probability [14], the inverse density function was calculated, clearing the basic density of the distribution function [13], which is the integral of function [12]. The values estimate for $\mu$ and $\sigma$ were 525.83 and 29.15 respectively.

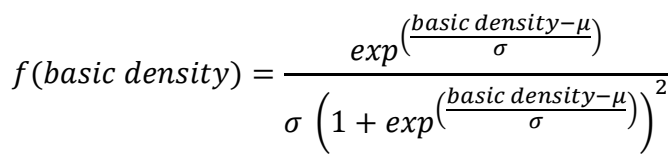

Probabity $=\frac{1}{1+\exp ^{-\left(\frac{\text { basic density- }}{\sigma}\right)}}$

basic density $=\mu+\sigma \ln \left(\frac{\text { probability }}{1-\text { probability }}\right) ; 0<$ probability $<1$

The quantification of the aerial biomass of each component on the 20 plots (Table 6) was made considering the selected functions (

\begin{tabular}{|c|c|c|c|c|c|c|c|c|c|c|c|}
\hline \multirow[b]{2}{*}{ : } & \multirow[b]{2}{*}{ } & \multicolumn{5}{|c|}{ Adjustment by location } & \multicolumn{5}{|c|}{ Adjustment for total locations } \\
\hline & & 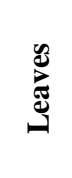 & 离 & 窇 & E & 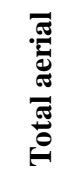 & 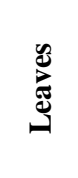 & 总 & 窇 & 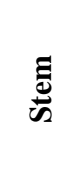 & 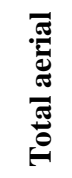 \\
\hline 1 & \multirow{8}{*}{$\begin{array}{l}\frac{\pi}{0} \\
\frac{0}{2} \\
\frac{0}{2}\end{array}$} & 0.0 & 0.0 & 0.0 & 22.8 & 25.5 & 4.0 & 2.8 & 12.4 & 23.7 & 27.7 \\
\hline 2 & & 4.6 & 0.8 & 1.7 & 7.3 & 5.3 & 6.0 & 2.1 & 14.8 & 8.2 & 5.9 \\
\hline 3 & & 1.8 & 2.0 & 1.6 & 0.1 & 0.2 & 18.9 & 6.4 & 14.5 & 7.1 & 5.0 \\
\hline 4 & & 6.2 & 2.0 & 3.6 & 0.0 & 0.0 & 19.2 & 4.8 & 16.0 & 16.2 & 14.2 \\
\hline 5 & & & & & & & 12.0 & 12.0 & 12.0 & 34.8 & 37.5 \\
\hline 6 & & & & & & & 16.6 & 12.8 & 13.7 & 19.3 & 17.3 \\
\hline 7 & & & & & & & 19.8 & 20.0 & 19.6 & 18.0 & 18.0 \\
\hline 8 & & & & & & & 24.2 & 20.0 & 21.6 & 18.1 & 18.2 \\
\hline 1 & \multirow{8}{*}{$\begin{array}{l}\frac{10}{0} \\
\stackrel{0}{0} \\
D \\
\infty\end{array}$} & 23.9 & 20.8 & 0.0 & 68.4 & 28.5 & 28.3 & 24.9 & 5.3 & 93.8 & 57.2 \\
\hline 2 & & 25.2 & 18.3 & 6.0 & 56.2 & 9.4 & 29.6 & 22.2 & 10.5 & 85.4 & 46.3 \\
\hline 3 & & 3.4 & 7.8 & 1.6 & 27.8 & 20.0 & 20.0 & 25.3 & 8.4 & 43.4 & 35.7 \\
\hline 4 & & 0.0 & 0.0 & 7.2 & 0.0 & 0.0 & 16.1 & 20.5 & 14.1 & 29.2 & 23.8 \\
\hline 5 & & & & & & & 35.9 & 32.8 & 12.0 & 80.4 & 40.5 \\
\hline 6 & & & & & & & 37.2 & 30.3 & 18.0 & 68.2 & 21.4 \\
\hline 7 & & & & & & & 21.4 & 25.8 & 19.6 & 45.8 & 38.0 \\
\hline 8 & & & & & & & 18.0 & 18.0 & 25.2 & 18.0 & 18.0 \\
\hline 1 & \multirow{8}{*}{$\begin{array}{l}\frac{8}{0} \\
\frac{\pi}{0} \\
0 \\
0 \\
0\end{array}$} & 0.0 & 14.1 & 10.7 & 29.3 & 18.8 & 5.9 & 19.5 & 27.8 & 63.1 & 45.1 \\
\hline 2 & & 0.6 & 11.0 & 5.5 & 19.2 & 0.9 & 9.4 & 15.1 & 26.5 & 43.3 & 19.8 \\
\hline 3 & & 1.3 & 1.9 & 3.4 & 28.0 & 20.6 & 4.7 & 21.5 & 27.3 & 44.7 & 38.1 \\
\hline 4 & & 2.4 & 0.0 & 0.0 & 0.0 & 0.0 & 8.1 & 16.6 & 24.3 & 6.9 & 7.2 \\
\hline 5 & & & & & & & 12.0 & 26.1 & 22.7 & 41.3 & 30.8 \\
\hline 6 & & & & & & & 12.6 & 23.0 & 17.5 & 31.2 & 12.9 \\
\hline 7 & & & & & & & 19.3 & 19.9 & 21.4 & 46.0 & 38.6 \\
\hline 8 & & & & & & & 20.4 & 18.0 & 18.0 & 18.0 & 18.0 \\
\hline 1 & \multirow{6}{*}{ 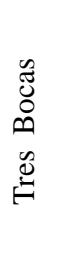 } & 3.0 & 1.6 & 0.1 & 17.0 & 12.9 & 6.5 & 12.4 & 1.7 & 30.5 & 28.2 \\
\hline 2 & & 0.0 & 0.0 & 0.0 & 12.3 & 4.3 & 2.7 & 9.6 & 1.0 & 24.7 & 20.5 \\
\hline 3 & & 4.6 & 2.6 & 1.4 & 8.7 & 10.8 & 9.1 & 14.7 & 3.9 & 16.5 & 20.2 \\
\hline 4 & & 1.4 & 0.4 & 1.3 & 0.0 & 0.0 & 5.8 & 12.4 & 3.3 & 8.1 & 10.8 \\
\hline 5 & & & & & & & 15.0 & 13.6 & 12.1 & 29.0 & 24.9 \\
\hline 6 & & & & & & & 12.0 & 12.0 & 12.0 & 24.3 & 16.3 \\
\hline
\end{tabular}

FLORESTA, Curitiba, PR, v. 50, n. 4, p. 1798 - 1807, out/dez 2020.

Vallejos-Barra, O. et.al.

ISSN eletrônico 1982-4688

1808

DOI: $10.5380 /$ rf.v50 i4. 65781 


\begin{tabular}{|c|c|c|c|c|c|c|c|c|c|c|c|}
\hline 7 & & & & & & & 22.6 & 20.6 & 19.4 & 26.7 & 28.8 \\
\hline 8 & & & & & & & 19.4 & 18.4 & 19.3 & 18.0 & 18.0 \\
\hline 1 & \multirow{8}{*}{ 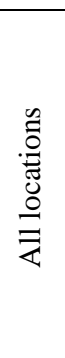 } & 41.5 & 48.0 & 6.2 & 141.6 & 94.3 & 58.3 & 71.5 & 41.7 & 215.2 & 176.7 \\
\hline 2 & & 44.5 & 40.4 & 9.2 & 98.4 & 28.5 & 60.4 & 59.4 & 46.1 & 172.3 & 118.4 \\
\hline 3 & & 4.4 & 13.7 & 0.0 & 50.6 & 53.9 & 49.5 & 72.6 & 42.7 & 97.1 & 107.5 \\
\hline 4 & & 0.0 & 0.0 & 5.0 & 0.0 & 0.0 & 42.2 & 57.5 & 45.4 & 62.8 & 61.7 \\
\hline 5 & & & & & & & 53.5 & 60.0 & 18.2 & 153.6 & 106.3 \\
\hline 6 & & & & & & & 56.5 & 52.5 & 21.2 & 110.4 & 40.5 \\
\hline 7 & & & & & & & 22.4 & 31.7 & 18.0 & 68.6 & 71.9 \\
\hline 8 & & & & & & & 18.0 & 18.0 & 23.0 & 18.0 & 18.0 \\
\hline
\end{tabular}

FLORESTA, Curitiba, PR, v. 50, n. 4, p. 1798 - 1807, out/dez 2020. 
Table 3). All functions are incorporated in DAP, in addition function [2] and [4] required the basic density, which was obtained by using a 50\% probability. Finally, with function [4] the tree heights had to be estimated as well, using the results of Table 5.

Table 6. Aerial biomass MAI estimation $\left(\mathrm{Mg} \mathrm{ha}^{-1} \mathrm{year}^{-1}\right)$ per location and component.

Tabela 6. Estimação do IMA da biomassa aérea $\left(\mathrm{Mg} \mathrm{ha}^{-1} \mathrm{año}^{-1}\right)$ por localidade e componente.

\begin{tabular}{cccccccc}
\hline Location & Age & N $\left(\right.$ Trees ha $\left.^{-\mathbf{1}}\right)$ & Leaves & Green branches & Dry branches & Stem & Total aerial \\
\hline Algorta & 4 & 1,267 & 1.038 & 1.234 & 1.031 & 12.881 & 16.195 \\
& 5 & 1,033 & 0.768 & 0.903 & 0.684 & 9.438 & 11.829 \\
& 8 & 1,033 & 0.681 & 0.784 & 0.472 & 8.455 & 10.415 \\
\hline Bequeló & 3 & 833 & 1.729 & 1.198 & 0.658 & 11.425 & 15.317 \\
& 4 & 1,033 & 1.590 & 1.122 & 0.630 & 12.139 & 15.863 \\
& 5 & 733 & 1.495 & 1.294 & 0.458 & 11.910 & 15.313 \\
& 6 & 1,233 & 1.496 & 1.139 & 0.566 & 13.240 & 16.674 \\
\hline Quebracho & 8 & 956 & 0.664 & 0.894 & 0.250 & 8.792 & 10.623 \\
\hline Tres Bocas & 9 & 1,133 & 0.988 & 1.188 & 0.453 & 12.890 & 15.563 \\
\hline
\end{tabular}

The validity of the analysis of parametric variance in the MAI for aerial biomass was checked, since both the assumption of normality (W-statistician of the Shapiro-Wilk test) and the assumption of homocedasticity (Zstatistician of the Levene test) were not statistically significant (Table 7). This analysis identified significant differences between the means of the locations in each of the components.

Table 7. Analysis of variance and Duncan's multiple range test of aerial biomass MAI ( $\left.\mathrm{Mg} \mathrm{ha}^{-1} \mathrm{año}^{-1}\right)$

Tabela 7. Análise de variância e proba de rangos múltiplos de Duncan do IMA da biomassa aérea $\left(\mathrm{Mg} \mathrm{ha}^{-1} \mathrm{ano}^{-1}\right)$

\begin{tabular}{|c|c|c|c|c|c|c|c|c|}
\hline \multicolumn{9}{|c|}{ Analysis of Variance } \\
\hline \multirow{2}{*}{$\begin{array}{l}\text { Component } \\
\text { Leaves }\end{array}$} & \multicolumn{2}{|c|}{$\mathbf{W}$} & \multicolumn{2}{|c|}{ 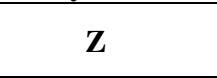 } & \multicolumn{2}{|c|}{$\begin{array}{c}\text { Value F of Factor } \\
\text { (Location) }\end{array}$} & \multicolumn{2}{|c|}{$\begin{array}{c}\text { Value F of covariate } \\
\text { (Trees ha-1) }\end{array}$} \\
\hline & 0.94 & (ns) & 2.84 & (ns) & 20.28 & $(* *)$ & 1.36 & (ns) \\
\hline Green branches & 0.96 & (ns) & 2.95 & (ns) & 3.81 & $(*)$ & 1.92 & (ns) \\
\hline Dry branches & 0.91 & (ns) & 2.37 & (ns) & 8.52 & $(* *)$ & 2.34 & (ns) \\
\hline Stem & 0.98 & (ns) & 0.75 & (ns) & 6.37 & $(* *)$ & 12.01 & $(* *)$ \\
\hline Total aerial & 0.98 & (ns) & 0.45 & (ns) & 12.56 & $(* *)$ & 9.14 & $(* *)$ \\
\hline
\end{tabular}

\begin{tabular}{|c|c|c|c|c|c|c|c|c|c|c|}
\hline \multicolumn{11}{|c|}{ Homogeneous locations per component according to Duncan's test $(* * *)$} \\
\hline Location & \multicolumn{2}{|c|}{ Leaves } & \multicolumn{2}{|c|}{ Green branches } & \multicolumn{2}{|c|}{ Dry branches } & \multicolumn{2}{|c|}{ Stem } & \multicolumn{2}{|c|}{ Total aerial } \\
\hline Algorta & 0.791 & $\mathrm{ab}$ & 0.921 & $\mathrm{a}$ & 0.621 & $\mathrm{~b}$ & 8.902 & a & 11.179 & a \\
\hline Bequeló & 1.623 & $\mathrm{c}$ & 1.213 & $\mathrm{ab}$ & 0.591 & $\mathrm{~b}$ & 12.149 & $\mathrm{~b}$ & 15.733 & $\mathrm{~b}$ \\
\hline Quebracho & 0.631 & a & 0.887 & $\mathrm{a}$ & 0.276 & $\mathrm{a}$ & 9.699 & $\mathrm{a}$ & 11.511 & $\mathrm{a}$ \\
\hline Tres Bocas & 1.072 & $\mathrm{~b}$ & 1.308 & $\mathrm{~b}$ & 0.444 & $a b$ & 12.021 & $\mathrm{~b}$ & 14.928 & $\mathrm{~b}$ \\
\hline
\end{tabular}

Where $(*)$ indicates significant statistical differences (probability $<0.05),(* *)$ indicates highly significant statistical differences (probability < 0.01 ), (ns) reveals that there are no significant differences (probability $\geq 0.05$ ). W is Shapiro-Wilk's statistician and $\mathrm{Z}$ is Levene's statistician. $\mathrm{F}$ corresponds to Snedecor's F-test statistician and $(* * *)$ indicates that there are no statistically significant differences between locations with the same sub-index letter in the vertical (probability $\geq 0.05$ ).

\section{DISCUSSION}

The increases presented in Table 1 are consistent with those reported by Resquin et al. (2012) for locations close to the geographical area of this research, so that the growth observed corresponds to the normal development of a plantation in these locations.

In Figure 1 total biomass and stem biomass are observed according to location. With this distribution of data, it would have been simpler to estimate the biomass of these components if the selected functions had considered the total of localities, since with one function the component under analysis would have been estimated. However, both adjustments for the total number of locations did not prove to be significant since the differences in the AIC, in most cases, were greater than 10 ( 
Table 2) and as indicated by Burnham and Anderson (2002) the use of these functions should be ruled out. locations (

In all components differences equal to zero were only found in the functions adjusted for each of the 
Table 2). This result coincides with that informed by Vallejos-Barra et al. (2014) in the estimation of absorbed carbon for the species and agrees with that expressed by van Breugel et al. (2011) and Paul et al. (2013).

$75 \%$ of the adjustments presented an adjusted $\mathrm{R}^{2}$ greater than 0.7 , showing a close relationship between the biomass and the explanatory variables considered. The contribution of the height variable was important since $60 \%$ of the selected functions incorporated this explanatory variable, which contradicts what was indicated by Montagu et al. (2005) and reinforces the points made by António et al. (2007). At the same time, the density of the wood was presented in $75 \%$ of the selected functions, in accordance with what was expressed by Kuyah et al. (2012); nevertheless, it was not possible to relate the density of the wood to the DBH, using a probability density function representing this variable.

The values of the universal exponent were lower than the theoretical value (8/3) in $70 \%$ of the selected functions, as was the case for Zianis and Mencuccini (2004), Pilli et al. (2006) and Kuyah et al. (2013).

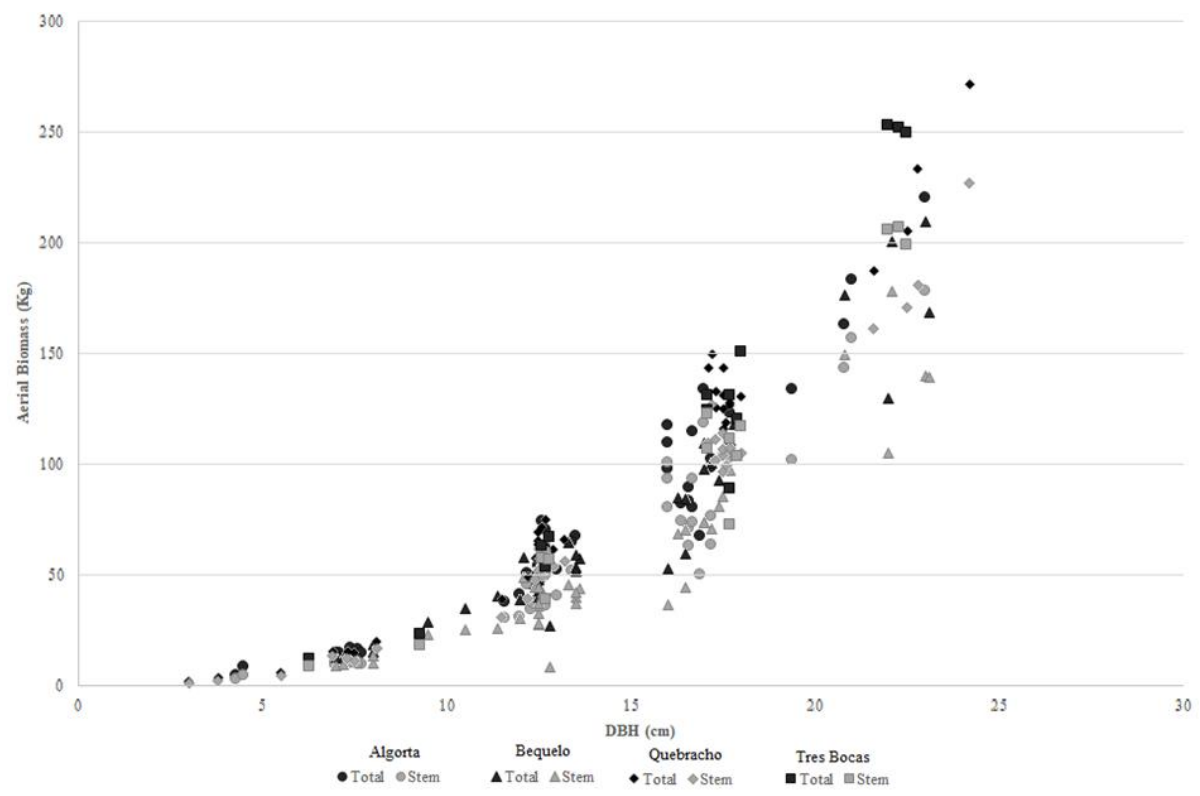

Figure 1. Total and stem biomass according to location

Figure 1. Biomassa total e de fuste segundo localidade

When determining the biomass for the components and their average annual increase (Table 6), the influence of the number of trees per hectare on the quantification of the aerial biomass of the stem and total is highlighted, due to the fact that the greatest increases are achieved with higher planting densities. On the other hand, in the remaining components, the greatest increases are frequently found in the lower planting densities.

The estimation of each component (Table 6 ) and its subsequent addition differs slightly from the estimates of the total by an average of $0.9 \%$ with a range that fluctuates between 0.1 and $2.4 \%$, confirming the consistency among the selected functions.

The recorded increases in the total aerial count coincide with those reported by Zewdie et al. (2009), not so in the remaining components, which evidences a differentiated distribution of biomass among the components between the two studies. Peréz-Cruzado and Rodriguez-Soalleiro (2011) obtained an increase in total biomass that fluctuated between 13.9 y $14.6 \mathrm{Mg} \mathrm{ha}^{-1} \mathrm{año}^{-1}$, which indicates some similarity with the results of this research.

The plantation density used as a covariate in the analysis of variance was highly significant for the total aerial and stem component, indicating that this covariate has direct implications for determining the increase in biomass for these components. In the remaining components there was no statistical significance, therefore, the number of trees per hectare considered in this research, did not significantly affect the increase in biomass of leaves, green branches and dry branches.

\section{CONCLUSIONS}

The analyzes carried out allow us to conclude that:

- The functions selected corresponded to those adjusted by location, described in Table 3 , since they presented the least difference from the AIC.

FLORESTA, Curitiba, PR, v. 50, n. 4, p. 1798 - 1807, out/dez 2020. 
- For the estimation of the stem and the total aerial the selected function is [4]. Leaves, green branches and dry branches in Algorta locality are estimated with function [1]; for these components in Bequeló and Quebracho function [1] or [4] should be used, as appropriate; on the other hand, in Tres Bocas the estimation of these components should be done with function [2].

- The global functions and the global functions that incorporated dummy variables showed an average difference from the AIC of 30.4, which makes their use impossible.

- The average annual increase of biomass presented statistical differences between the localities conformed by two groups, the one with the highest increase represented by Bequeló and Tres Bocas (15.733 y $14.928 \mathrm{Mg}$ $\mathrm{ha}^{-1}$ year $^{-1}$ respectively), the other made up of Quebracho and Algorta (11.511 y $11.179 \mathrm{Mg} \mathrm{ha}^{-1}$ year $^{-1}$ respectively).

\section{REFERENCES}

ÁlvareZ, E.; DUQUE, A.; SALDARRIAGA, J.; CABRERA, K.; DE LAS SALAS, G.; DEL VALLE, I.; LEMA, A.; MORENO, F.; ORREGO, S.; RODRÍGUEZ, L. Tree above-ground biomass allometries for carbon stocks estimation in the natural forests of Colombia. Forest Ecology and Management v. 267, p. 297-308, 2012.

ANTÓNIO, N.; TOMÉ, M.; TOMÉ, J.; SOARES, P.; FONTES, L. Effect of tree, stand, and site variables on the allometry of Eucalyptus globulus tree biomass. Canadian Journal of Forest Research. v. 37, n. 5, p. 895-906, 2007.

BURNHAM, K. P.; ANDERSON, D. R. Model selection and multimodel inference. A practical informationTheoretic Approach. Second Edition. Springer. 2002, 515 p.

BURNHAM, K. P.; ANDERSON, D. R.; HUYVAERT, K. P. AIC model selection and multimodel inference in behavioral ecology: some background, observations, and comparisons. Behav Ecol Sociobiol v. 65, p. 23-35, 2011.

CHAVE, J.; ANDALO, C.; BROWN, S.; CAIRNS, M.A.; CHAMBERS, J.Q.; EAMUS, D.; FOLSTER, H.; FROMARD, F.; HIGUCHI, N.; KIRA, T.; LESCURE, J.P.; NELSON, B.W.; OGAWA, H.; PUIG, H.; RIÉRA, B.; YAMAKURA, T. Tree allometry and improved estimation of carbon stocks and balance in tropical forests. Oecologia v. 145, n. 1, p. 87-99, 2005.

CRUZ, G.; MUNKA, C.; PEDOCCHI, R. Regionalización agroclimática del litoral centro - oeste del Uruguay. Agrociencias. v. 4, n. 1, p. $87-92,2000$.

DGF (Dirección General Forestal) - MGAP (Ministerio de Ganadería. Agricultura y Pesca). Bosques plantados registrados. Eucalyptus globulus ssp. globulus. <http://www2.mgap.gub.uy/portal/page.aspx?2,dgf,dgf-recurso-forestal,O,es,0,.> Acceso 03/08/2017.

GIBBS, H.; BROWN, S.; NILES, J.; FOLEY, J. A. Monitoring and estimating tropical forest carbon stocks: making REDD a reality. Environ. Res. Lett. v. 2, p. 1 -13, 2007.

INIA. Caracterización Agroclimática del Uruguay. 1980-2009. Serie Técnica 193, 2011, 34 p.

KUYAH, S.; DIETZ, J.; MUTHURI, C.; JAMNADASS, R.; MWANGI, P.; COE, R.; NEUFELDT, H. Allometric equations for estimating biomass in agricultural landscapes: I. Aboveground biomass. Agriculture. Ecosystems and Environment v. 158, p. 216 - 224, 2012.

KUYAH, S.; DIETZ, J.; MUTHURI, C.; VAN NOORDWIJK, M.; NEUFELDT, H. landscapes. Biomass and bioenergy v. 55, p. $276-284,2013$.

MAZEROLLE, M. J. Improving data analysis in herpetology: using Akaike's Information Criterion (AIC) to assess the strength of biological hypotheses. Amphibia-Reptilia v. 27, p. 169-180, 2006.

MONTAGU, K.; DÜTTMER, K.; BARTON, C.; COWIE, A. Developing general allometric relationships for regional estimates of carbon sequestration - an example using Eucalyptus pilularis from seven contrasting sites. Forest Ecology and Management v. 204, p. 113-127, 2005.

PAUL, K.I.; ROXBURGH, S. H.; RITSON, P.; BROOKSBANK, K.; ENGLAND, J. R.; LARMOUR, J. S.; RAISON, R.J.; PECK, A.; WILDY, D. T.; SUDMEYER, R. A.; GILES, R.; CARTER, J.; BENNETT, R.; MENDHAM, D. S.; HUXTABLE, D.; BARTLE, J. R. Testing allometric equations for prediction of above-ground biomass of mallee eucalypts in southern Australia. Forest Ecology and Management v. 310, p. 1005-1015, 2013.

FLORESTA, Curitiba, PR, v. 50, n. 4, p. 1798 - 1807, out/dez 2020.

Vallejos-Barra, O. et.al.

ISSN eletrônico 1982-4688

1813

DOI: $10.5380 /$ rf.v50 i4. 65781 
PÉREZ-CRUZADO, C.; RODRÍGUEZ-SOALLEIRO, R. Improvement in accuracy of aboveground biomass estimation in Eucalyptus nitens plantations: Effect of bole sampling intensity and explanatory variables. Forest Ecology and Management v. 261, n. 11, p. 2016-2028, 2011.

PILLI, R.; ANFODILLO, T.; CARRER, M. Towards a functional and simplified allometry for estimating forest biomass. Forest Ecology and Management v. 237, p. 583-593, 2006.

RESQUIN, F.; FARIÑA, I.; RACHID, C.; RAVA, A.; DOLDÁN, J. Influencia de la edad de corte en el pulpeo de Eucalyptus globulus plantado en Uruguay. Agrociencia Uruguay v, 16, n. 2, p. 27-38, 2012.

SNIPES, M.; TAYLOR, D. C. Model selection and Akaike Information Criteria: An example from wine ratings and prices. Wine Economics and Policy v. 3, p. 3-9, 2014.

VALLEJOS-BARRA, O.; DANILUK-MOSQUERA, G.; MORAS, G.; PONCE-DONOSO, M.; CONTRERAS, M. A. Above-ground carbon absorption in young Eucalyptus globulus plantations in Uruguay. Sci. For. Piracicaba. v. 42, n. 101, p. 9-19, 2014.

VAN BREUGEL, M.; RANSIJN, J.; CRAEN, D.; BONGERS, F.; HALL, J. Estimating carbon stock in secondary forests: decisions and uncertainties associated with allometric biomass models. For. Ecol. Manage. v. 262, p. 1648-1657, 2011.

WANG, J.; ZHANG, C.; XIA, F.; ZHAO, X.; WU, L.; VAN GADOW, K. Biomass structure and allometry of Abies nephrolepis (Maxim) in Northest China. Silva Fennica v. 45, n. 2, p. 211-226, 2011.

ZENG, W. S.; ZHANG, H. R.; TANG, S.Z. Using the dummy variable model approach to construct compatible single-tree biomass equations at different scales - a case study for Masson pine (Pinus massoniana) in southern China. Canadian Journal of Forest Research v. 41, n. 7, p. 1547-1554, 2011.

ZEWDIE, M.; OLSSON, M.; VERWIJST, T. Above-ground biomass production and allometric relations of Eucalyptus globulus Labill. Coppice plantations along a chronosequence in the central highlands of Ethiopia. Biomass Bioenergy v. 33, n. 3, p. $421-428,2009$

ZIANIS, D.; MENCUCCINI, M. On simplifying allometric analyses of forest biomass. Forest Ecology and Management v. 187, p. 311-332, 2004.

ZIANIS, D.; XANTHOPOULOS, G.; KALABOKIDIS, K.; KAZAKIS, G.; GHOSN, D.; ROUSSOU, O. Allometric equations for aboveground biomass estimation by size class for Pinus brutia Ten. trees growing in North and South Aegean Islands. Greece. Eur J Forest Res v. 130, p. 145-160, 2011. 